\title{
Lymph Node Staging as Haruspication
}

\author{
Clifford S. Cho, MD FACS (1) \\ Department of Surgery, University of Michigan Medical School, Ann Arbor, MI
}

According to those with knowledge of antiquity, ${ }^{1}$ it was once accepted practice to inspect animal (and, on occasion, human) entrails to predict the future. In Mesopotamian, Etruscan, Hittite, and Ancient Roman societies, highly trained individuals now referred to as seers and priests harvested, prepared, and arranged explanted livers and intestines in hopes of identifying anatomic harbingers of fortune or doom for patients stricken with illness. This practice, apparently called haruspication, was occasionally used to develop more global predictions for weather, politics, and society. In this current era of oncology, highly trained individuals now referred to as pathologists and surgical oncologists harvest, prepare, and arrange explanted lymphatic tissues in hopes of identifying anatomic harbingers of fortune or doom for patients with resected cancer. This practice, called nodal staging, is occasionally used to develop more global prognostication systems for large populations of patients.

In the current issue of Annals of Surgical Oncology, Chen and coauthors, representing an impressive multicenter Chinese hepatobiliary clinical research collaborative, report a methodical study of 226 patients with resected gallbladder carcinoma. This dataset allows them to ask a question that has been lobbed back and forth between investigators and journals over the past decade: What is the best way to quantify the predictive impact of nodal status? Put another way, what is the best way to interpret the fortune-telling power lurking within resected lymph nodes? This investigative endeavor has deployed serious mathematical and scientific firepower over the years. It was once

(C) Society of Surgical Oncology 2021

First Received: 16 August 2021

Accepted: 18 August 2021;

Published Online: 2 September 2021

C. S. Cho, MD FACS

e-mail: cliffcho@med.umich.edu enough to know the number of lymph nodes that harbored metastatic disease. Then, accounting for the invisible influences of understaging (inadequate nodal yield), it was suggested that the ratio of positive to negative lymph nodes was more informative. Then, to better capture the probabilities that metastases are present or not present within a sampled set of lymph nodes, odds ratios (conventionally transformed into logarithmic scale to better illustrate positive versus negative likelihoods) were proffered as a way to quantify the prognostic information hidden within lymph nodes.

The previous paragraph may merit further exploration for those of us who do not reflexively view the world through mathematical concepts beyond basic arithmetic operations. Consider a situation in which a person's entire hepatobiliary nodal drainage system contains 8 lymph nodes, 2 of which harbor metastatic foci of gallbladder carcinoma (Fig. 1). The well-meaning hepatobiliary surgeon skeletonizes this person's porta hepatis, removing all visible nodal tissues, and in the process removes 5 lymph nodes. Following careful microscopic haruspication, as far as the person, surgeon, and collaborating pathologist are aware, there exist 5 lymph nodes, 1 of which harbors a metastatic focus of gallbladder carcinoma and 4 of which are completely benign. The number of metastatic lymph nodes (NMLN) (1) offers meaningful prognostic insight, as this perceived NMLN of 1 predicts something very different than what might be expected if the number were 0 . However, both the surgeon and pathologist harbor a sneaking suspicion that additional lymph nodes (perhaps hidden behind the portal vein, or along the common hepatic artery) may yet lurk unresected within the person's upper abdomen; indeed, unbeknownst to them, the actual NMLN is 2. Thus, in an effort to account for the possibility that additional lymph nodes (and lymph node metastases) might have been missed, the lymph node ratio (LNR) of positive lymph nodes divided by total number of lymph nodes $(1 / 5$, or 0.2) softens the impact of a value as unsubtle and 


\begin{tabular}{|c|c|}
\hline $\begin{aligned} \text { perceived reality }= & 1 \text { metastatic lymph node } \\
& 4 \text { normal lymph node }\end{aligned}$ & $\begin{aligned} \text { actual reality }= & 2 \text { metastatic lymph node } \\
& 6 \text { normal lymph node }\end{aligned}$ \\
\hline perceived $\mathrm{NMLN}=1$ & actual NMLN $=2$ \\
\hline perceived $\mathrm{LNR}=0.2$ & actual $\mathrm{LNR}=0.25$ \\
\hline perceived $\mathrm{LODDS}=-0.47712$ & actual LODDS $=-0.47712$ \\
\hline
\end{tabular}

FIG. 1 Three ways to measure the difference between perception and reality in nodal staging

artificially definitive as the NMLN of 1 . Indeed, the difference between the perceived $L N R$ of 0.2 and the actual $L N R$ of 0.25 feels less dramatic than the difference between the perceived NMLN of 1 and the actual NMLN of 2 . However, the surgeon and pathologist both recognize that things could have been worse; had the surgeon removed only 4 lymph nodes and missed the one that had metastatic disease, both the NMLN and the LNR would have been the same: 0 . What would have happened if the surgeon had only resected 3 lymph nodes? Or 2? The haunting recognition of the potential shortcomings of NMLN and LNR motivates them to rely not on the counting of positive lymph nodes, but on the probability that positive lymph nodes might have been found. The logarithmic odds of positive lymph nodes (LODDS) use the data available to the surgeon and pathologist to ask the following question: If only one lymph node had been harvested, what is the probability that that lymph node would have harbored metastatic disease? More specifically, it measures the ratio of two counterfactual probabilities - the probability that the one lymph node would have been positive and the probability that the one lymph node would have been negativeand transforms it into logarithmic scale to turn the value into a digestible value, with positive values representing an increased chance of finding metastasis and negative values representing a decreased chance. In this scenario, the perceived LODDS based on available data is -0.47712 , indicating that, if only one lymph node had been harvested, it would probably have been negative; and, indeed, in this scenario, the actual LODDS is also -0.47712 , narrowing the gap between perception and reality.

Many academic papers and promotions have been generated through exhaustive comparisons of these three methodologies, applied to many different kinds of cancers-far too many to cite. As might be expected, there has been a general trend favoring LNR over NMLN, and a general (but more subtle) trend favoring LODDS over LNR. However, these trends are far from uniform; hence, the present study from Chen and colleagues.

So what do they find? Applying sophisticated mathematical methods with names like tree-augmented naïve Bayesian modeling, they conclude that, for patients with resected gallbladder carcinoma, NMLN, LNR, and LODDS compete like three world-class athletes in an Olympic sprint. If not for the second-splitting accuracy of our measurement technology, it would have been a tie; but because of our ability to calculate accuracy, concordance index (c-index), and area under the curve (AUC) to two and three decimal points, the winner is: our old friend NMLN.

The margin by which NMLN wins this race is truly razor-thin and of questionable statistical significance: NMLN outperformed LNR and LODDS by 0.81 and $2.96 \%$ respectively in accuracy, by 0.004 and 0.015 respectively in c-index, and by 0.000 and 0.023 respectively in AUC. Nevertheless, we disembark from this whirlwind journey with two sinking feelings: First, that after having expended so much effort and creativity and math, we have ended up in exactly the same place whence we began; and second, that after splitting so many hairs and leaving so few stones unturned, this metaphorical horse may finally be too moribund to submit to further punishment.

There comes a time in any human endeavor when the right thing to do is to move on. Very few journeys, whether actual or scientific, end up not having been worth their while. While comparing methods of lymph node staging, many lessons of intellectual humility, curiosity, and insight were learnt along the way. Yet it does feel as though we have finally reached the point where the methodology and utility of lymph node haruspication has become as optimized as they need to be. Lymph node metastases are prognostically significant. The assessment of lymph node metastases has been and will remain subject to biases of understaging. All of this is practically informative and intellectually interesting, but what we need to do now is to go back to answering the harder questions: How do metastases form? Where along the process of metastasis does lymphatic dissemination occur?

Thankfully, some of the most intelligent people in this world are asking and, incrementally, answering these questions. The scientific literature is replete with rigorously proven insights and brave speculations that are mind-altering in ways that nodal staging analysis is not. Did you know that, as cancer cells begin to first take root in the pancreas, the liver begins to alter its environment in ways 
that eventually make it much more hospitable to future metastatic travelers? ${ }^{2}$ Maybe distant organs are not innocent victims of but knowing accessories to the crime of metastasis. Have you heard that cancer cells exposed to lymph are protected from mechanisms of immune-mediated cell death in ways that cancer cells exposed to blood are not? $?^{3,4}$ Maybe cancer cells are not infiltrating into lymph nodes because they have suddenly acquired intrinsic properties of enhanced aggressiveness but merely doing so to escape harsher environments. Thanks to the work of investigators such as Chen and coauthors, perhaps it is now time to move on from seeking haruspicatory signs that predict the future, to understanding the events that cause it.

\section{REFERENCES}

1. https://futurescopes.com/divination/haruspication/2316/haruspicati on-predicting-future-inspecting-entrails-animal

2. Lee JW, Stone ML, Porrett PM, et al. Hepatocytes direct the formation of a pro-metastatic niche in the liver. Nature. 2019;567:249-52.

3. Ubellacker JM, Tasdogan A, Ramesh V, et al. Lymph protects metastasizing melanoma cells from ferroptosis. Nature. 2020;585:113-8.

4. Wang W, Green M, Choi JE, et al. CD8(+) T cells regulate tumour ferroptosis during cancer immunotherapy. Nature. 2019;569:270-4.

Publisher's Note Springer Nature remains neutral with regard to jurisdictional claims in published maps and institutional affiliations. 